\title{
Development
}

\section{Does Birth Trigger Cell Death in the Developing Brain?}

\author{
Alexandra Castillo-Ruiz, ${ }^{1}$ Taylor A. Hite, ${ }^{1}$ Dina W. Yakout, ${ }^{1}$ T. John Rosen, ${ }^{2}$ and Nancy G. Forger ${ }^{1}$
}

https://doi.org/10.1523/ENEURO.0517-19.2020

${ }^{1}$ Neuroscience Institute, Georgia State University, Atlanta, GA 30302 and ${ }^{2}$ Department of Mathematics and Statistics, Georgia State University, Atlanta, GA 30302

\section{Visual Abstract}

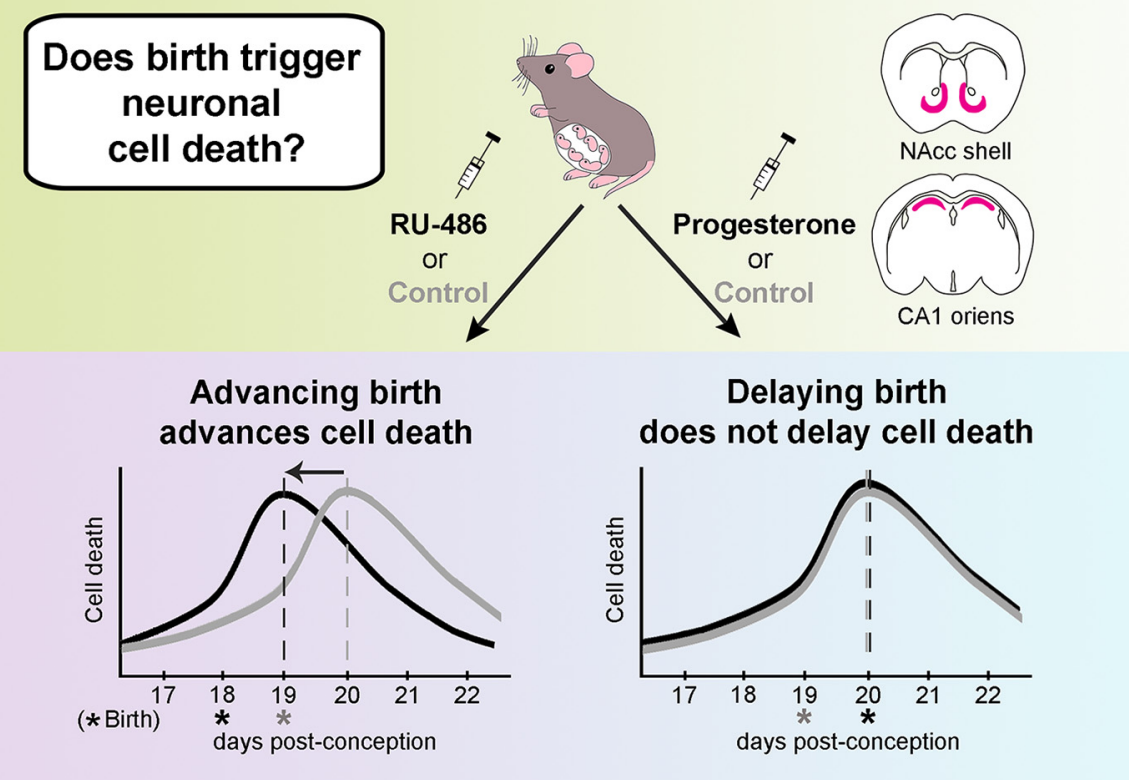

Developmental cell death eliminates half of the neurons initially generated in the mammalian brain, and occurs perinatally in many species. It is possible that the timing of neuronal cell death is developmentally programmed, and only coincidentally associated with birth. Alternatively, birth may play a role in shaping cell

\section{Significance Statement}

The importance of neuronal cell death for brain development has been recognized for decades, but it is unknown what regulates its timing, or accounts for differences in the amount of cell death between brain regions. In many species, including mice, developmental cell death occurs perinatally. We find that advancing birth by $1 \mathrm{~d}$ in mice advances patterns of cell death, but does not advance overall forebrain (FB) growth. Because humans across the world routinely alter birth timing, usually to advance birth, our findings may have implications for current obstetric practices. Birth timing also affects the magnitude of cell death in a region-specific manner, suggesting that birth has important, previously unrecognized, effects on brain development. 
death. To test these competing hypotheses, we experimentally advanced or delayed birth by $1 \mathrm{~d}$ in mice (within the normal range of gestation for the species) and examined effects on the temporal pattern and magnitude (amount) of neuronal cell death, using immunohistochemical detection of activated caspase-3 as a cell death marker. In order to detect effects of subtle changes in birth timing, we focused on brain areas that exhibit sharp postnatal peaks in cell death. We find that advancing birth advances peak cell death, supporting the hypothesis that birth triggers cell death. However, a delay of birth does not delay cell death. Thus, birth can advance cell death, but if postponed, a developmental program governs. Advancing or delaying birth also caused region-specific changes in the overall magnitude of cell death. Our findings shed light on the longstanding question of what controls the timing and magnitude of developmental neuronal cell death, and position birth as an orchestrator of brain development. Because humans across the world now routinely alter birth timing, these findings may have implications for current obstetric practices.

Key words: apoptosis; birth; birth timing; cell death; cesarean; parturition

\section{Introduction}

Developmental cell death is an important feature of brain development in all vertebrates. In mammals, approximately $50 \%$ of the neurons initially generated are eliminated during a discrete perinatal period (Oppenheim, 1991; Dekkers et al., 2013). Developmental neuronal cell death occurs by apoptosis, and culminates in the activation of caspases, including caspase-3, which causes cellular demise through the cleavage of key intracellular proteins (Porter and Jänicke, 1999; Hengartner, 2000). Although the importance of this process has been recognized for over 50 years, many basic questions still remain. It is unknown, for example, what initiates or terminates the cell death period. Specifically, does the timing of neuronal cell death reflect intrinsic factors (i.e., an autonomous developmental program), or do extrinsic factors play a role? Also, what accounts for the large (>10-fold; Ahern et al., 2013) regional differences in the magnitude of cell death? The tight association of cell death with birth in mice provides an opportunity to address these questions.

A cell death "atlas" of the mouse brain was recently compiled, using immunohistochemistry for activated caspase-3 (AC3) to quantify apoptosis in over 20 forebrain (FB) regions prenatally and postnatally (Ahern et al., 2013; Mosley et al., 2017). Although AC3 has been reported in the absence of apoptosis, the large majority of cells

Received December 8, 2019; accepted January 15, 2020; First published February 3, 2020.

The authors declare no competing financial interests.

Author contributions: A.C.-R. and N.G.F. designed research; A.C.-R., T.A.H., and D.W.Y. performed research; A.C.-R. and T.J.R. analyzed data; A.C.-R. and N.G.F. wrote the paper.

This work was supported by the National Science Foundation Grant IOS1743673 (to N.G.F.).

Acknowledgements: We thank Geert J. de Vries, William J. Schwartz, and Carla D. Cisternas for helpful comments on the manuscript; Jeff Reese and Naoko Boatwright for advice on manipulating gestation length; and Morgan M. Mosley, Jennifer Gray, and Andrew J. Jacobs for technical assistance.

Correspondence should be addressed to Nancy G. Forger at nforger@ gsu.edu.

https://doi.org/10.1523/ENEURO.0517-19.2020

Copyright (C) 2020 Castillo-Ruiz et al.

This is an open-access article distributed under the terms of the Creative Commons Attribution 4.0 International license, which permits unrestricted use, distribution and reproduction in any medium provided that the original work is properly attributed. positive for AC3 in the perinatal rodent brain co-label with other cell death markers, and are neurons (Srinivasan et al., 1998; Zuloaga et al., 2011; Ahern et al., 2013). Results of the atlas study show that the cell death period is largely compressed into the first postnatal week in mice, with the highest number of dying cells seen during the first $3 \mathrm{~d}$ after birth in many brain regions (Ahern et al., 2013; Mosley et al., 2017). One possible explanation for these findings is that extrinsic factors associated with labor, parturition, and/or the transition to ex utero life may determine cell death timing. If so, then advancing or delaying birth would be expected to advance or delay cell death, respectively. Alternatively, if the timing of cell death is based on an autonomous developmental program, then patterns would depend on postconceptional age, independent of birth timing.

To distinguish between these hypotheses, we manipulated birth timing in C57BL/6 mice. Most births in this strain occur at $19 \mathrm{~d}$ postconception (dpc; Murray et al., 2010), but a range of $18-20 d$ is considered normal (Donahue, 2012). We randomly assigned timed-pregnant dams to give birth early (18 dpc), on-time (19 dpc), or late (20 dpc) within this range. Parturition in mice is normally triggered when the corpus luteum regresses, leading to an abrupt fall in progesterone (Ratajczak and Muglia, 2008; Mitchell and Taggart, 2009). Although there are several ways to control parturition timing (e.g., ovariectomy, gene knock-outs), the approach we took here comes closest to replicating how short and long gestations naturally vary from each other, i.e., we mimicked the hormonal profiles of an "advanced" or "delayed" parturition by injecting a progesterone receptor antagonist to advance birth, or by clamping progesterone levels to delay birth (Dudley et al., 1996; Hashimoto et al., 2010).

In order to be able to discern effects of a subtle change in gestation length (i.e., $1 \mathrm{~d}$ ), we required brain regions with discrete neonatal peaks in cell death, and large changes in apoptosis from one day to the next. The cell death atlas study described above allowed us to identify two brain regions fulfilling these criteria: the CA1 oriens layer of the hippocampus (CA1or) and the shell of the nucleus accumbens (NAcc). Both of these regions show a sharp increase in cell death in the $24 \mathrm{~h}$ after birth, followed by a rapid decline over the next 2 d (Ahern et al., 2013; Mosley et al., 2017).

We reasoned that if neuronal cell death is independent of birth, then patterns will be determined by postconceptional 
age, regardless of when pups are delivered. Alternatively, if birth plays an important role in triggering cell death, patterns will be determined by days postnatal, independent of days postconception. We find that advancing birth advances cell death patterns, suggesting that an early birth can trigger cell death. In contrast, cell death depends on postconceptional age if birth is delayed. We also find that birth timing influences the magnitude of cell death in a brain region-specific manner.

\section{Materials and Methods}

\section{Animals and timed pregnancies}

Adult female and male C57BL/6 mice were obtained from our breeding program or purchased from The Jackson Laboratory. Mice were maintained on a 12/12 h light/dark cycle with ad libitum access to food and water. All animal procedures were performed in accordance with the Georgia State University animal care committee's regulations. We established timed-pregnancies by pairing animals within an hour of lights off and removing males 1$2 \mathrm{~h}$ after lights on. The morning after pairing was considered embryonic day $0(0 \mathrm{dpc})$ and cages were checked four to six times a day for births.

\section{Advancing birth}

To advance birth, we injected pregnant dams at $17 \mathrm{dpc}$ with RU-486 (150 $\mu \mathrm{g}$ in 0.1-ml sesame oil; Sigma-Aldrich; catalog \#M8046; $N=7$ ), as previously described (Dudley et al., 1996; Toda et al., 2013). RU-486 is an antagonist of both progesterone and glucocorticoid receptors (Baulieu, 1989). When administered to the mother, it is detected in the fetal circulation (albeit at much lower levels) and does not affect estradiol, glucocorticoid, or progesterone levels in the mother or the fetus (Wolf et al., 1988; Hill et al., 1990). Injections were administered five to 10 min before lights off, and controls received vehicle injections $(N=9)$ on the same schedule. All RU-486-treated dams gave birth on $18 \mathrm{dpc}$, within $18 \mathrm{~h}$ of treatment. Pups of both sexes were weighed, euthanized, and their brains collected on postnatal day (P)0 (the day of birth), P1, and P2 (between 9 and $11 \mathrm{~h}$ after lights-on each day). During the course of this study, one unmanipulated timed-pregnant dam from our breeding colony and three vehicle-treated dams also gave birth on $18 \mathrm{dpc}$. As there were no differences in cell death between the offspring delivered on $18 \mathrm{dpc}$ "spontaneously" or after RU-486 treatment (Extended Data Fig. 1-1), data from all advanced births were pooled in the analyses below.

\section{Delaying birth}

To delay birth, we injected dams with progesterone (0.5-1 mg in 0.1- to 0.2-ml vehicle; Sigma Aldrich; catalog \#P3972; $N=23$ ), as per (Hashimoto et al., 2010) on 17 and $18 \mathrm{dpc}, 5 \mathrm{~h}$ after lights on. Controls received the vehicle ( $2 \%$ ethanol in peanut or sesame oil; $N=5)$. Most $(N=12$; $52 \%$ ) of the progesterone-treated dams gave birth on 20 $\mathrm{dpc}$, and the offspring of eight of these pregnancies were used in the main experiment. Two treated dams (9\%) gave birth on $19 \mathrm{dpc}$ and nine (39\%) gave birth on $21 \mathrm{dpc}$; a subset of the pups born on $21 \mathrm{dpc}$ (from five different litters) were also examined, as described below. Births tended to occur within $6 \mathrm{~h}$ after lights on, and pups of both sexes were weighed, euthanized, and their brains collected on P0, P1, and P2 (between 9 and $11 \mathrm{~h}$ after lights on each day).

\section{Effects of RU-486 and progesterone on cell death in utero}

To test for effects of RU-486 and progesterone on neuronal cell death in the offspring in utero, timed-pregnant dams were treated as above. Three hours after the last injection, dams were anesthetized with $2 \% \mathrm{CO}_{2}$ and rapidly decapitated. The fetuses were removed from the uterine horns, and their brains collected and processed for the detection of AC3. We also assessed progesterone levels in the offspring of progesterone- and vehicle-treated dams by ELISA. Trunk blood was collected and plasma from two to six embryos was pooled to generate each sample. Samples were analyzed in duplicate at the University of Virginia Center for Research in Reproduction Ligand Core Laboratory according to the manufacturer's recommendations (Immuno-Biological Laboratories Inc.).

\section{Immunohistochemistry}

Brains were immersion fixed in $5 \%$ acrolein for $24 \mathrm{~h}$, followed by $30 \%$ sucrose for at least $48 \mathrm{~h}$, and then transferred to cryoprotectant solution at $-20^{\circ} \mathrm{C}$ until sectioning. Brains were coronally frozen-sectioned into four, $40-\mu \mathrm{m}$ series, and two series were used for the immunohistochemical detection of dying cells, as previously (Ahern et al., 2013; Mosley et al., 2017; Castillo-Ruiz et al., 2018a,b). Briefly, epitope retrieval and unreacted aldehyde blockade was performed with $30-\mathrm{min}$ incubations in $0.05 \mathrm{M}$ sodium citrate and $0.1 \mathrm{M}$ glycine, respectively. Tissue was then incubated in a blocking solution $(20 \%$ normal goat serum and $1 \%$ hydrogen peroxide) for $30 \mathrm{~min}$, followed by an overnight incubation in AC3 primary antibody (Cell Signaling; catalog \#9661, RRID:AB_2341188; $1: 20,000$ for newborns and 1:10,000 for fetuses). On the following day, the tissue was incubated with goat antirabbit secondary antibody (Vector Laboratories; catalog \#BA-1000, RRID:AB_2313606; 1:500) for $1 \mathrm{~h}$, and then with avidin and biotin (Vector Laboratories; catalog \#PK$6100 ; 1: 500)$ for $1 \mathrm{~h}$. The tissue was reacted for $30 \mathrm{~min}$ with 3,3'-diaminobenzidine tetrahydrochloride, nickel sulfate, and hydrogen peroxide. Tissue was mounted onto gelatin-coated slides, counterstained with thionin, and coverslipped.

\section{Quantification of AC3 labeling}

For analysis of cell death in newborn mice, slides were scanned using a Hamamatsu Nanozoomer (Hamamatsu Photonics K.K.) and AC3 + cell quantification was performed using Aperio Image Scope (Leica Biosystems Inc). For the smaller experiments in which cell death was examined in fetuses, AC3 immunostaining was quantified live on a microscope using Stereo Investigator software (MBF Biosciences). In both cases, we drew contours 
around the areas of interest and AC3 + cells within each contour were quantified as previously described (Ahern et al., 2013; Mosley et al., 2017; Castillo-Ruiz et al., 2018a,b). Because AC3 cells are relatively sparse, all positive cells could be counted and random sampling was not required. Cells were counted only when the cell body was clearly visible within the section. The sum of AC3 + cell counts across all sections was divided by total area sampled, and then multiplied by section thickness to obtain the density of AC3+ cells per $\mathrm{mm}^{3}$ for each animal. Investigators blind to experimental conditions performed all analyses.

\section{Brain regions and total $F B$ volume}

For the CA1or, our sampling spanned the dorsal hippocampus. We analyzed sections starting where the dentate gyrus makes its rostral-most appearance (Paxinos et al., 2007, their Fig. 65 of the developing mouse brain atlas), and ending when the lateral extent of the hippocampus starts to tip ventrally (Paxinos et al., 2007, their Fig. 70 of the developing mouse brain atlas) as described in (Mosley et al., 2017); in general, this included four alternate sections per animal. For the shell of the NAcc, we included sections starting at the point at which the corpus callosum joins at midline (Paxinos et al., 2007, their Fig. 54 of the developing mouse brain atlas) to the point where the anterior commissure starts elongating horizontally (Paxinos et al., 2007, their Fig. 58 of the developing mouse brain atlas); in general, this included six alternate sections per animal. We estimated overall brain size by outlining the left side of the entire FB from the point where the medial border of the anterior commissure is located ventral to the tip of the lateral ventricle to the rostral most appearance of the dorsomedial nucleus of the hypothalamus (Paxinos et al., 2007, their Figs. 57-70 of the developing mouse brain atlas) as described previously (Castillo-Ruiz et al., 2018a).

\section{Statistical analyses}

Statistical analyses were performed using IBM SPSS Statistics v.25 (IBM Corp.) and GraphPad Prism v.8 (GraphPad Software, Inc.). We first performed threeway ANOVAs (group $\times$ postnatal age $\times$ sex) for all dependent measures (i.e., cell death, FB size, and body weight). No significant effects of sex were identified for any measure; the data from males and females therefore were pooled and two-way ANOVAs (group $\times$ postnatal age, OR group $\times$ postconceptional age) were used in the statistical analyses. Because changing the timing of birth could have influenced the pattern and/or the magnitude of cell death, we evaluated these possibilities separately. Cell death pattern matching between treatment groups was performed by converting AC3 data to Z-scores, with mean and standard error calculated per treatment group; this eliminated group differences due only to magnitude. The magnitude of cell death was then compared between treatment groups using the raw scores. Main effects and significant interactions were followed by Bonferroni tests. Two-tailed independent $t$ tests were used to test for the acute effects of RU-486 or progesterone treatment in fetuses.

\section{Results}

\section{Advancing birth by $1 \mathrm{~d}$ advances cell death}

Neuronal cell death was compared on P0, P1, and P2 in mice born "on time" or $1 \mathrm{~d}$ early (Fig. $1 A ; N=12-20$ mice per group). This corresponded to 19, 20, and $21 \mathrm{dpc}$ for all on-time births, and 18, 19, and $20 \mathrm{dpc}$ for "advanced births" (Fig. 1A). Birth timing could affect the temporal pattern of cell death, the overall magnitude (amount), or both. To test for effects on the pattern, independent of changes in magnitude, AC3 cell densities were transformed to Z-scores (within treatment group) and doubleplotted to contrast the competing hypotheses, i.e., with either postnatal age or postconception age on the $x$-axis (Fig. $1 B, C$ ). If birth triggers cell death, then cell death patterns would be similar between groups when plotted as a function of postnatal age. On the other hand, if cell death is developmentally programmed, then the pattern of cell death should depend on postconceptional age, regardless of day of birth.

The prediction that birth timing determines cell death timing was supported. In the CA1or and NAcc, a prominent peak in cell death was previously reported $1 \mathrm{~d}$ after birth $(P 1=20$ dpc; Mosley et al., 2017). Here, peak cell death again occurred $1 \mathrm{~d}$ after birth $(\mathrm{P} 1)$ in both brain regions and for both groups, despite the fact that this was $19 \mathrm{dpc}$ for the advanced-birth group and $20 \mathrm{dpc}$ for the on-time birth group (Fig. 1B-E). In agreement, two-way ANOVA found no differences in cell death between birth groups when analyzed with respect to postnatal age in either the CA1or or NAcc. In contrast, when the data were analyzed as a function of postconception age, there was a significant interaction between days postconception and group in both regions (CA1or: $F_{(1,59)}=38.14, p<$ 0.0001 ; NAcc: $\left.F_{(1,59)}=16.45, p=0.0001\right)$, reflecting the fact that peak cell death occurred on different days postconception for the groups (Fig. 1B,C). [Note: the ANOVAs for postnatal age included all time points (P0, P1, P2) and those for postconception age included only timepoints for which both groups were represented (19 and $20 \mathrm{dpc}$ ).]

\section{Advancing birth decreases overall cell death in the NAcc}

Next, to determine whether birth timing affected the magnitude of cell death independent of the temporal pattern, we calculated the main effect of group on cell death across all three postnatal days, this time using the raw number of dying cells ( $N=38-52$ mice per group). Advancing birth did not alter the magnitude of cell death in the CA1or (Fig. 1F). However, overall cell death in the advanced birth group was reduced by approximately $33 \%$ in the NAcc compared with controls $\left(F_{(1,84)}=16.09\right.$, $p=0.0001$; Fig. $1 F$.

\section{RU-486 does not alter cell death independent of effects on birth timing}

The fact that cell death patterns in RU-486-treated offspring did not differ from those of neonates spontaneously delivered on $18 \mathrm{dpc}$ (Materials and Methods; Extended Data Fig. 1-1) supports the conclusion that 
A

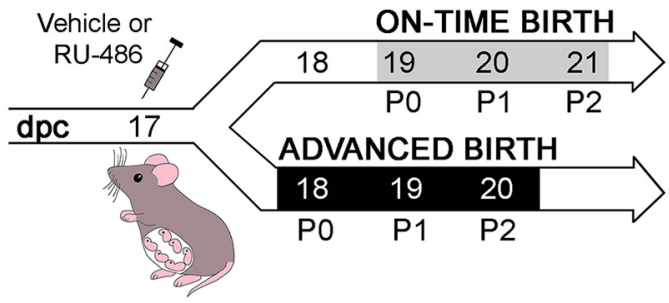

B

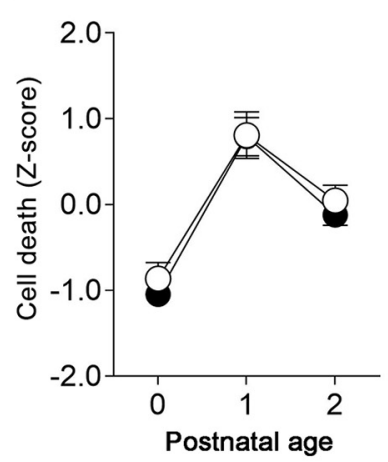

C
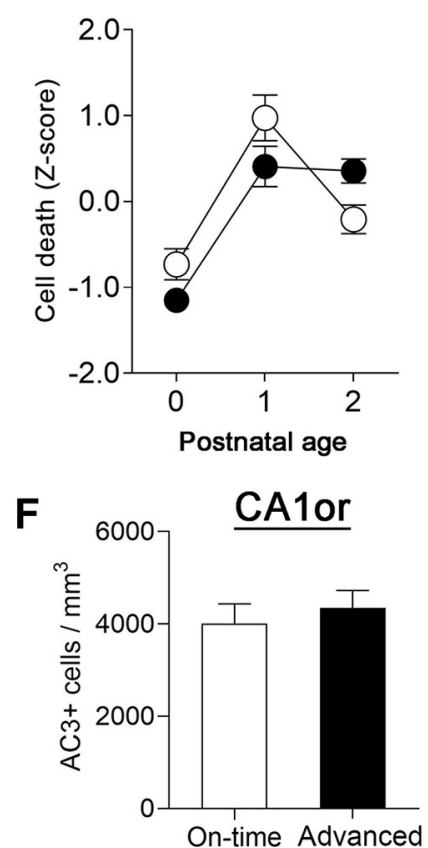

\section{CA1or}

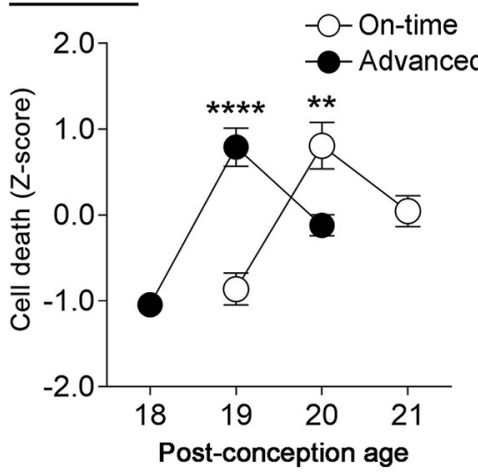

\section{NAcC}
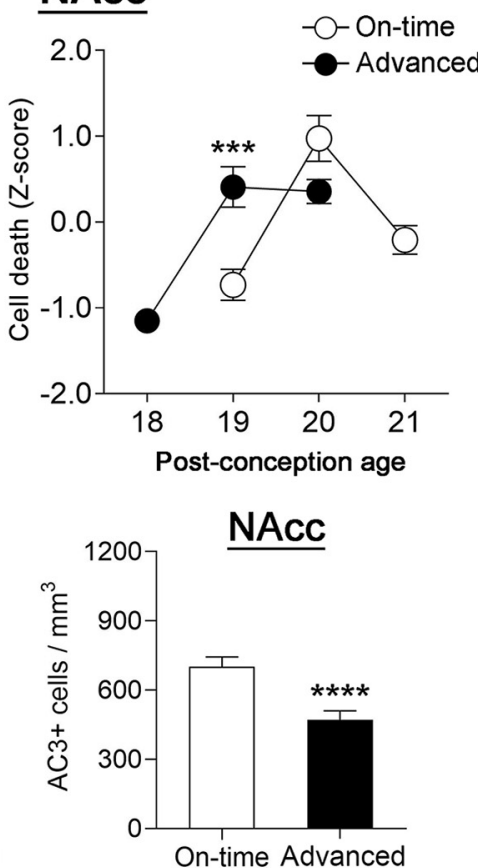

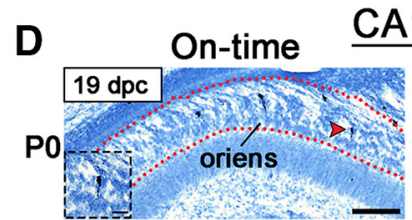

A10r Advanced
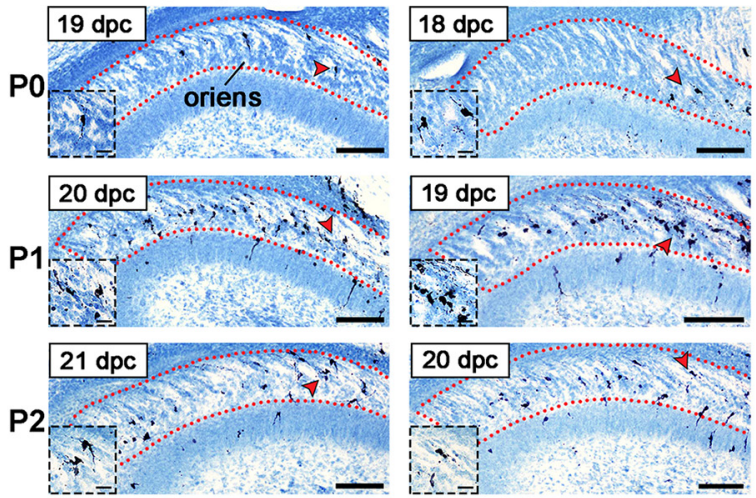

E
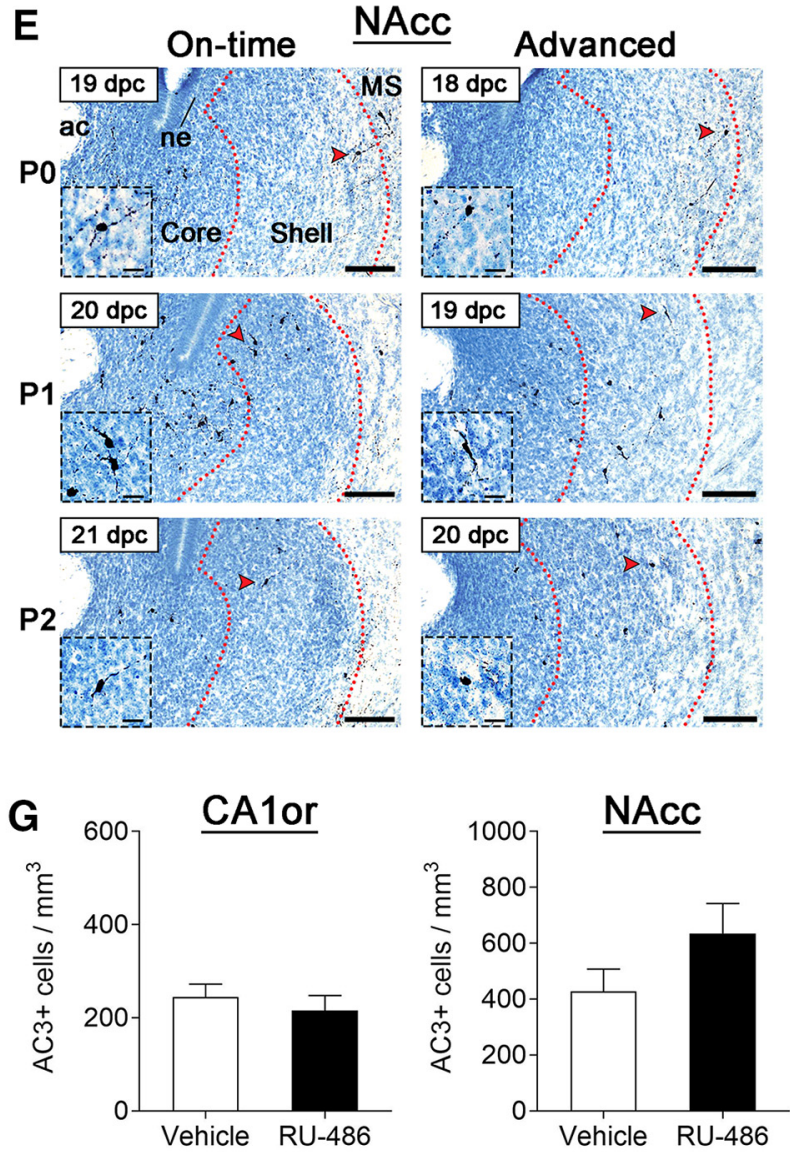

Figure 1. Advancing birth by $1 \mathrm{~d}$ advances cell death. $\boldsymbol{A}$, Timeline of experimental procedures. $\boldsymbol{B}, \boldsymbol{C}$, Cell death was very similar between the on-time and advanced groups when compared by postnatal age (left), but not by postconception age (right) in both the CA1or and NAcc. Asterisks indicate significant differences by Bonferroni post hoc tests: $* * p<0.01, * * * p<0.001, * * * * p<0.0001$. In addition, cell death did not differ between pups with spontaneous advanced birth versus those with advanced birth after RU-486 treatment of the dam (Extended Data Fig. 1-1). D, E, Photomicrographs of AC3-labeled tissue (counterstained with thionin) in representative sections from the on-time and advanced birth groups in the CA1or and NAcc. Arrowheads point to cells shown at higher magnification in the insets. Peak cell death occurred at P1, despite the fact that this was $20 \mathrm{dpc}$ for on-time births and $19 \mathrm{dpc}$ for advanced births; ac, anterior commissure; MS, medial septum; ne, neuroepithelium. Scale bars $=100 \mu \mathrm{m}$ (main photograph) and $20 \mu \mathrm{m}$ (insets). $\boldsymbol{F}$, Advancing birth by a day did not change the magnitude of cell death over the first three postnatal days in the CA1or, but decreased cell death in the NAcc; $* * * p=0.0001$. G, RU-486 did not alter cell death in fetuses $3 \mathrm{~h}$ after treatment of the dam in either the CA1or or NAcc. Means \pm SEM are plotted for all figures except for $\boldsymbol{F}$, which shows estimated marginal means \pm SEM. 
A

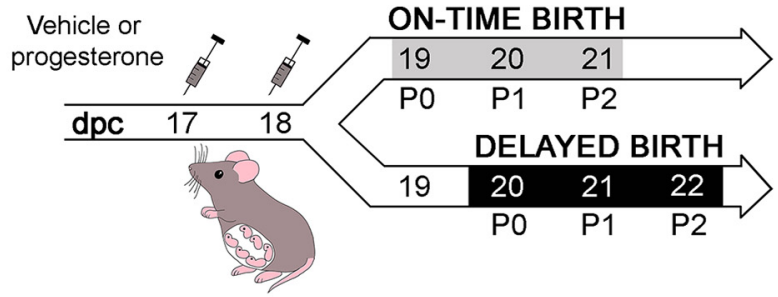

B

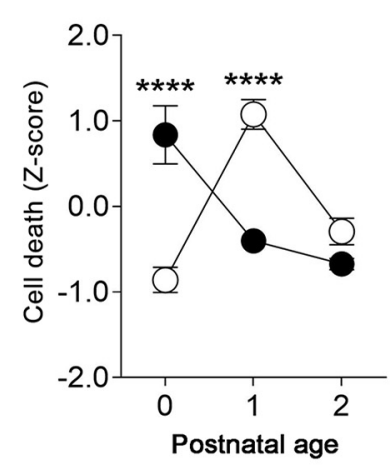

C

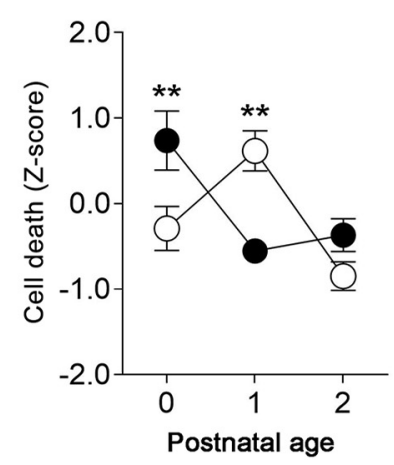

$\mathbf{F}$

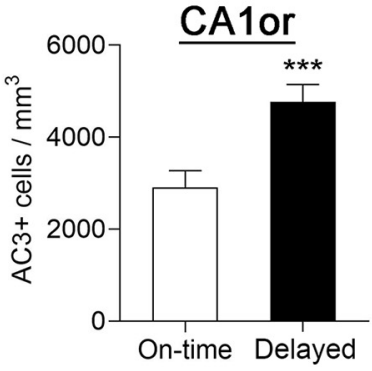

CA1or

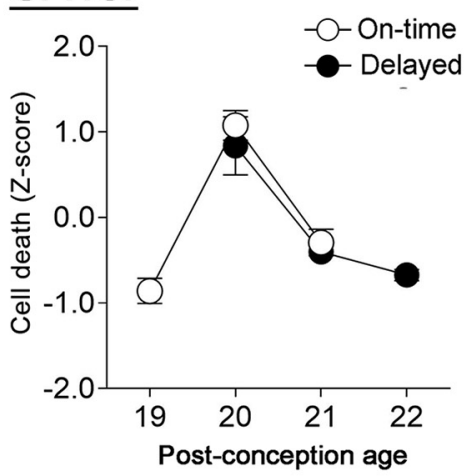

NAcC
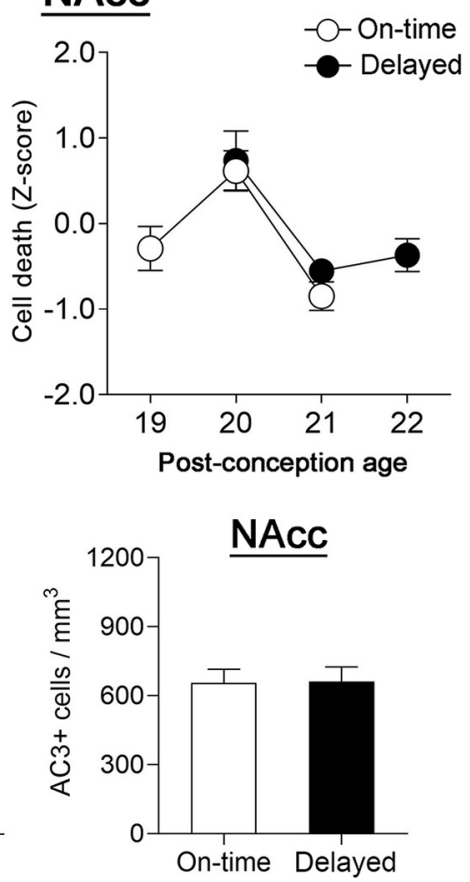

D

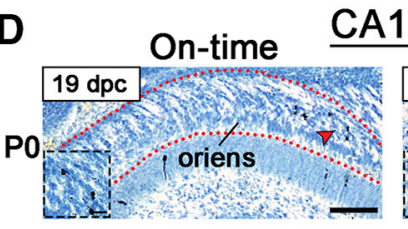

CA1or

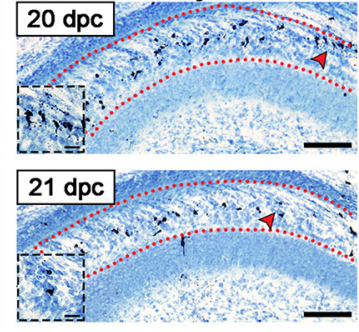

P1

$20 \mathrm{dpc}$
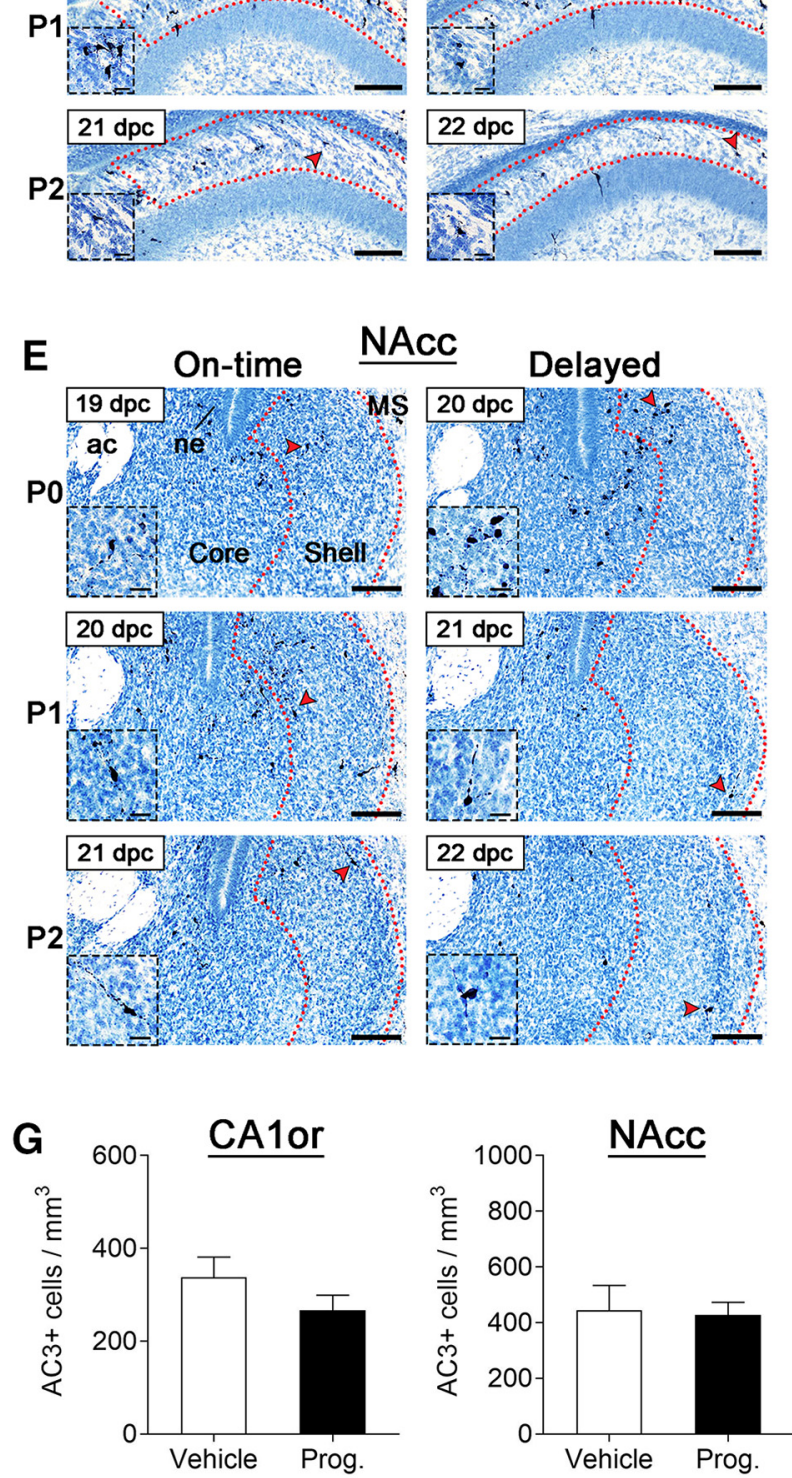

Figure 2. Delaying birth by a day does not delay cell death. $\boldsymbol{A}$, Timeline of experimental procedures. $\boldsymbol{B}, \boldsymbol{C}$, Patterns of cell death in on-time versus delayed birth groups best match when plotted as a function of postconception age (right), rather than postnatal age (left) in both the CA1or and NAcc. Asterisks indicate significant differences on each day by Bonferroni tests: $* * p<0.01, * * * * p<$ 0.0001. D, E, Photomicrographs of AC3-labeled tissue (counterstained with thionin) in representative sections of mice from the ontime and delayed birth groups in the CA1or and NAcc. Arrowheads point to cells shown at higher magnification in the insets. Peak cell death occurred at $20 \mathrm{dpc}$, despite the fact that this was P1 for the on-time birth group and P0 for the delayed-birth group; ac, anterior commissure; MS, medial septum; ne, neuroepithelium. Scale bars $=100 \mu \mathrm{m}$ (main photograph) and $20 \mu \mathrm{m}$ (insets). $\boldsymbol{F}$, Delaying birth by a day increased cell death magnitude overall in the CA1or but did not affect the magnitude of cell death in the NAcc; $* * * p=0.0007$. G, Progesterone treatment of the dams increased progesterone levels in fetuses (as described in Results) but did not affect cell death in either brain region. Means \pm SEM are plotted for all figures except for $\boldsymbol{F}$, which shows estimated marginal means \pm SEM. 
changes in birth timing, rather than some other effect of $\mathrm{RU}-486$, caused an advance in cell death. To further assess whether RU-486 treatment of dams alters neuronal cell death in their offspring independent of birth, a new cohort of timed-pregnant dams was treated with vehicle or RU-486, as above (Fig. 1A). When administered to the mother, RU-486 is found in the fetal circulation within a few hours (Wolf et al., 1988; Hill et al., 1990). We therefore collected the brains of offspring $3 \mathrm{~h}$ after injection $(N=12-$ 17 mice per group), while all offspring were still in utero, and processed them for the detection of AC3. We found no effect of RU-486 treatment on cell death in utero in either the CA1 or or NAcc (Fig. 1G).

\section{Delaying birth by $1 \mathrm{~d}$ does not delay cell death}

If advancing birth advances cell death, a delay in birth may delay cell death. Alternatively, endogenous signals might trigger cell death when birth is delayed. To test this, we treated timed-pregnant dams with progesterone on 17 and $18 \mathrm{dpc}$ to delay parturition (Hashimoto et al., 2010) by a day; i.e., birth occurred on $20 \mathrm{dpc}$ ("delayed birth" group). Their offspring were compared with those of vehicle controls which gave birth at $19 \mathrm{dpc}$ (on-time birth). The brains of offspring were again examined on P0, P1, and P2 ( $N=9-12$ mice per group), which in this case corresponded to 19,20 , and $21 \mathrm{dpc}$ for the on-time group and 20, 21, and $22 \mathrm{dpc}$ for the delayed birth group (Fig. $2 A)$.

Contrary to what was seen with advancing birth, postconceptional age, rather than postnatal age, best predicted cell death patterns when birth was delayed. For both the CA1or and NAcc, the peak of cell death occurred at $20 \mathrm{dpc}$, despite the fact that this was $\mathrm{P} 1$ for the on-time group and $\mathrm{PO}$ for the delayed group (Fig. $2 B-E$ ). Two-way ANOVAs (group $\times$ postnatal age) confirmed a significant interaction in both regions (CA1or: $F_{(2,58)}=35.42, p<$ 0.0001; NAcc: $\left.F_{(2,60)}=11.43, p<0.0001\right)$, due to the fact that peak cell death occurred on different days postnatal (Fig. 2B,C). In contrast, when the data were analyzed as a function of postconceptional age, the two-way ANOVA found no differences between the groups and no group $x$ age interaction (Fig. 2B,C). Further supporting these findings, we examined cell death in the offspring of five progesterone-treated dams that gave birth on $21 \mathrm{dpc}$ (i.e., 2 $\mathrm{d}$ after expected delivery). Again, cell death was higher on P0 than on P1 in the CA1or and NAcc (Fig. 3), which is predicted if cell death is driven by a developmental program.

\section{Delaying birth increases overall cell death in the CA1or}

To determine whether a delay in birth altered overall cell death magnitude, we calculated the main effect of group on AC3 cell number across all three postnatal ages. A delay in birth increased cell death magnitude overall by approximately $64 \%$ in the $\mathrm{CA} 1$ or $\left(F_{(1,58)}=12.72, p=\right.$ 0.0007 ), but had no effect in the NAcc (Fig. 2F). Thus, delaying birth does not delay the timing (i.e., temporal
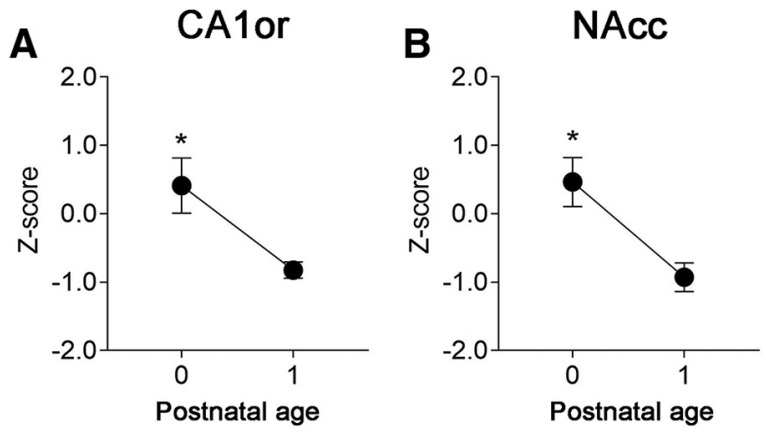

Figure 3. Cell death declines between $\mathrm{P0}$ and $\mathrm{P} 1$ in pups born 2 d late. Cell death in the CA1or $(\boldsymbol{A})$ and NAcc $(\boldsymbol{B})$ was higher on $\mathrm{P} 0$ than on $\mathrm{P} 1$ ( $p=0.04$ and $p=0.02$, respectively) in pups born $2 \mathrm{~d}$ late (on $21 \mathrm{dpc}$ ) to dams treated with progesterone on days 17 and 18 postconception. This outcome is in contrast to that seen for mice born on-time (where cell death in the CA1 and NAcc is low on P0 and peaks on P1; Figs. 1, 2B,C) but is expected if a developmental program governs cell death when birth occurs late; $N=3-6$ mice per group. Means \pm SEM are plotted, ${ }^{*} p<0.05$.

pattern) of developmental cell death, but may cause region-specific differences in magnitude.

\section{Progesterone treatment does not acutely alter cell death}

To determine whether progesterone treatment of dams alters cell death of fetuses in utero, a separate cohort of timed-pregnant dams was treated with progesterone or vehicle on 17 and $18 \mathrm{dpc}$ (as above; Fig. $2 A$ ). The trunk blood and brains of offspring were collected $3 \mathrm{~h}$ after the last injection ( $27 \mathrm{~h}$ after the first injection) to measure circulating progesterone ( $N=7-9$ pooled samples per group) and AC3-cell number ( $N=12-26$ mice per group), respectively. As expected, progesterone levels were significantly increased in the offspring of treated dams (3.67 vs $29.19 \mathrm{ng} / \mathrm{ml}$ for vehicle and progesterone-treated samples; $p=0.0002$ ). However, there was no effect on cell death in either the CA1 or or NAcc (Fig. 2G).

\section{Overall brain growth is determined by days postconception}

It was possible that the advance of cell death patterns following an early birth was an indirect effect of an overall acceleration in brain development. To address this, we calculated total FB volume for all animals in the advanceand delay-birth experiments ( $N=9-20$ mice per group). Interestingly, FB volume depended on postconceptional age regardless of whether birth was advanced or delayed. When analyzed with respect to postnatal age, there were highly significant group differences in FB size between on-time and advanced births $\left(F_{(1,81)}=69.06, p<0.0001\right.$; Fig. $4 A$, left), as well as between on-time and delayed births $\left(F_{(1,60)}=56.91, p<0.0001\right.$; Fig. $4 B$, left). By contrast, when analyzed by postconceptional age, there was no difference in FB size between advanced birth and ontime groups (Fig. $4 A$, right). There was a subtle effect of group when birth was delayed $\left(F_{(1,41)}=9.68, p=0.0034\right.$; 

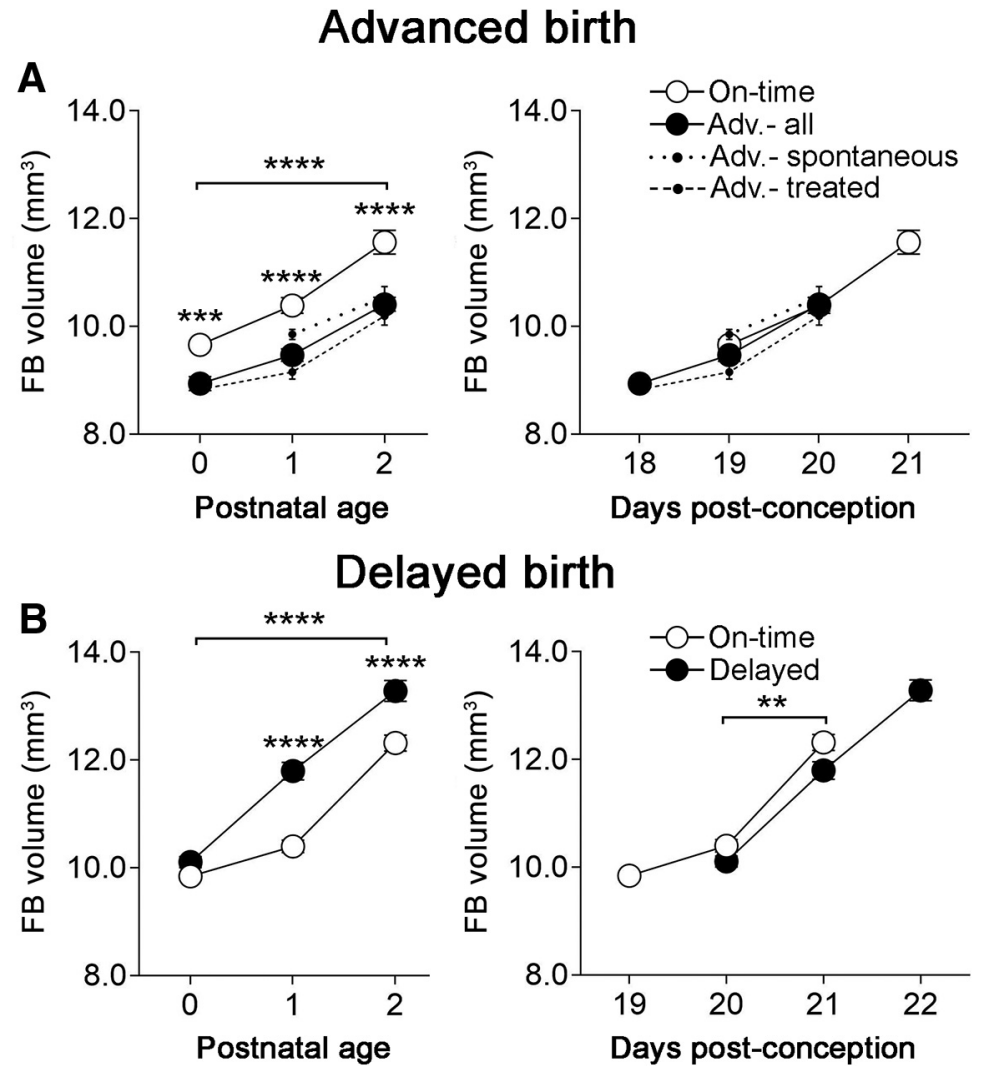

Figure 4. Overall brain growth is determined by postconceptional age. FB growth was best predicted by postconception age (right), rather than postnatal age (left) whether birth was advanced $(\boldsymbol{A})$ or delayed $(\boldsymbol{B})$. Because mice with spontaneous advanced births differed from mice with advanced birth after RU-486 treatment on this measure, the data for those groups are plotted separately (dotted and hashed lines, respectively), as well as combined (solid lines). However, the comparison between on-time and advanced groups remained significant whether considering spontaneous only, treated only, or all advanced births ( $p<0.0001$ in each case). Asterisks indicate significant differences on each day by Bonferroni post hoc tests. Brackets indicate main effect of group. $* * p<0.01, * * * p=0.001, * * * * p<0.0001$. Means \pm SEM are plotted.

Fig. $4 B$, right), although the difference was not significant for any single age in the post hoc tests.

Thus, a generalized advance of brain development is unlikely to account for our cell death results (above) and, as a result, cell death decouples from overall brain development in animals experiencing advanced birth. The temporal pattern of cell death was also dissociated from overall body growth. Body weight lagged behind that of controls at all postnatal ages in the advancedbirth group, and was predicted by postnatal age, rather than postconceptional age, following a delayed birth (Fig. 5).

\section{Discussion}

Although neuronal cell death clusters around the time of birth in many species, a causal relationship between birth and cell death has not been probed. Indeed, it is tricky to do so: manipulations of birth timing must be made within a narrow time window in order to avoid possible confounds associated with prematurity and postmaturity, and this, in turn, requires the identification of brain regions with striking day to day changes in cell death. Using quantitative cell death data from a previous study to identify appropriate brain regions and an approach that mimics natural hormonal variations around the time of delivery in mice, we found that advancing birth advances patterns of developmental neuronal cell death. However, if birth is delayed, cell death occurs on time. These findings therefore support both of our original hypotheses: environmental signals can determine cell death timing, but in the absence of those signals a developmental program governs.

One limitation of the current study is that we focused our analyses on two brain regions with relatively sharp postnatal peaks in apoptosis. On the one hand, this was a strategic choice that allowed us to test our hypotheses. However, it is possible that our results are not representative of all brain regions if, for example, we unintentionally selected for regions sensitive to birth timing. In fact, we attempted to quantify cell death in several other regions in the material at hand, but results were inconclusive, as it is difficult to clearly discern the effect of a 1-d change in gestation length in brain regions that do not exhibit a sharp peak in cell death. It is possible that with a very large number of animals per group, or in longer-lived species with more protracted cell death periods, this could eventually be addressed. Nonetheless, this study provides proof-of-concept that extrinsic factors such as birth can drive cell death patterns and, in so doing, enables us 

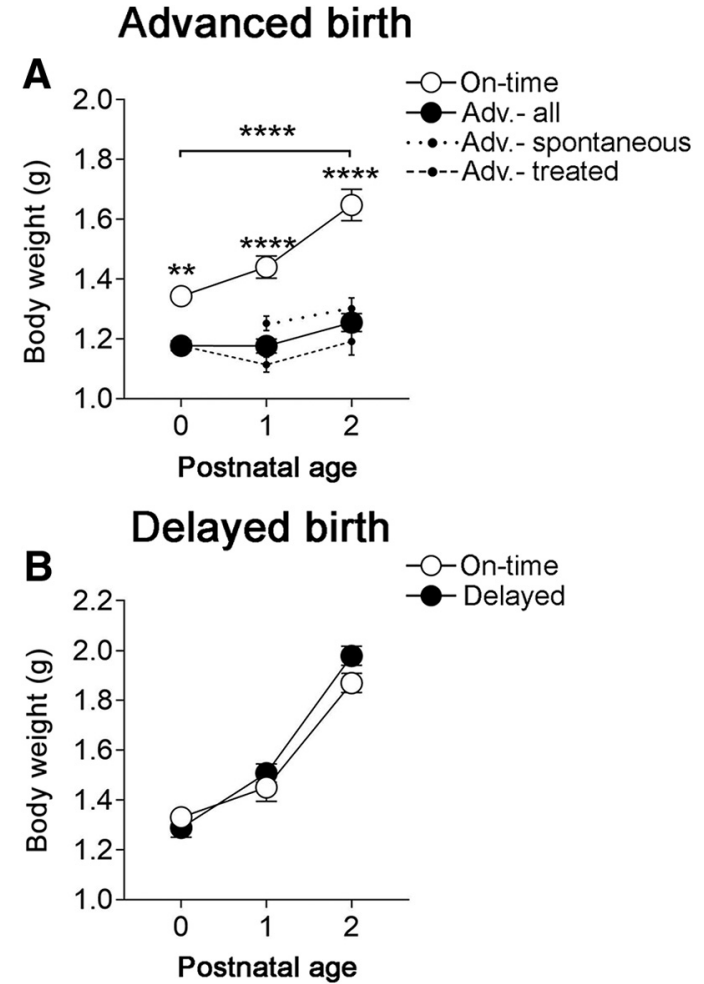

Figure 5. Effects of an advanced or delayed birth on overall body growth. $\boldsymbol{A}$, When birth was advanced (filled circles), body growth lagged behind that of pups delivered on-time (open circles) for all postnatal ages $\left(F_{(1,83)}=98.16, * * * * p<0.0001\right.$ for main effect of group; brackets). Because mice with spontaneous advanced births (dotted lines) differed from mice with advanced birth after RU-486 treatment (hashed lines) on this measure, the data for those groups are plotted separately. However, the comparison between on-time and advanced groups remains significant whether considering spontaneous only, treated only, or all advanced births (solid line, $p<0.0001$ in each case). Asterisks indicate significant differences on each day by Bonferroni tests: $* * p<0.01$, $* * * * p<0.0001$. $\boldsymbol{B}$, In contrast, body weight did not differ following an on-time versus delayed delivery $\left(F_{(1,60)}=1.62, p=0.21\right) ; N=9-20$ mice per group. Means \pm SEM are plotted.

to address a long-standing question about the timing of developmental neuronal cell death.

Another potential concern is that our treatments themselves may have influenced cell death. It is reassuring that there was no difference in cell death in either the CA1 or $\mathrm{NAcc}$ in animals born early spontaneously versus after RU486 treatment. We also did not find evidence for an acute change in cell death in either brain region after RU-486 or progesterone treatment of the dams, and progesterone was clearly elevated in fetuses at the timepoint sampled. However, we examined only a single, relatively early timepoint in each case. Although steroid hormones can affect cell death quite rapidly (Mann et al., 2000; Estrada et al., 2006; Waters and Simerly, 2009; Morrissy et al., 2010), we cannot rule out the possibility that we missed effects that may have occurred at a time not sampled here.

"Birth" is a complex stimulus, accompanied by marked hormonal changes, the mechanical stimuli associated with labor and delivery, and many physiological changes required for the fetus to survive ex utero. Which of these factors is responsible for advancing cell death patterns following an early birth remains to be determined. In addition to the obligatory drop in progesterone, a vaginal birth triggers an "adaptive stress response" which leads to marked increases in vasopressin, glucocorticoids, and catecholamines in the offspring (for review, see Lagercrantz and Slotkin, 1986; Evers and Wellmann, 2016). Neuronal cell death can be curbed or promoted by each of these hormones (Noh et al., 1999; Mulholland et al., 2005; Noguchi et al., 2008; Chen and Aguilera, 2010; Zuloaga et al., 2011), and receptors for them are widespread across the perinatal brain (Yi et al., 1994; Happe et al., 2004; Quadros et al., 2007, 2008; Hammock, 2015). Thus, it is possible that hormonal factors associated with labor or parturition may play a role in triggering cell death when birth is advanced. In addition, birth is an inflammatory event, marked by a surge in proinflammatory cytokines in amniotic fluid and a peripheral immune response in the newborn (Osman et al., 2003; Golightly et al., 2011; Walker et al., 2011; Kaiser et al., 2014; Kingsbury and Bilbo, 2019), which could also influence neuronal cell death. It may be possible in future studies to manipulate individual hormones or immune signaling molecules to test their roles in the control of cell death.

To our knowledge, only one other study has examined how a change in birth timing affects a well-charted neurodevelopmental event. Toda and colleagues found that when birth was advanced by $1 \mathrm{~d}$ in mice, the initiation of barrel formation in somatosensory cortex was accelerated (Toda et al., 2013; delayed birth was not examined in this study). This effect was related to the fact that birth triggers a decrease in extracellular serotonin, which permits barrel formation (Toda et al., 2013). Together with the present results, this suggests that an advanced birth may organize multiple events across the developing brain. However, effects are specific because an early birth does not accelerate overall brain development. In fact, global measures such as total FB volume in the current study, cortical thickness in the Toda et al. study (Toda et al., 2013), and brain weight in pigs (Holme Nielsen et al., 2018), all depend on postconceptional and not postnatal age.

In contrast to the effects of advancing birth, a delay of birth did not delay cell death patterns, supporting the existence of a developmental program. In a related study, Southwell et al. (2012) transplanted cortical interneurons from 13.5- or 14.5-dpc donor mice into the brains of older hosts. Because the transplanted neurons underwent cell death at a time consistent with the age of the donor, rather than of the host, the authors concluded that interneuron cell death is determined by the intrinsic maturational state of the neurons (Southwell et al., 2012). Genetic and molecular regulators that could underlie such a developmental timing program have been well characterized in invertebrates (Grosskortenhaus et al., 2005; Moss, 2007), but are less studied in vertebrates. Our current data support the existence of an intrinsic developmental program, but one that can be accelerated by an advanced birth. 
We also found effects of birth timing on overall magnitude of cell death in the neonatal brain, with an early birth decreasing cell death in the NAcc (but not CA1or) and a late birth increasing cell death in the CA1or (but not the NAcc). Because these effects were in opposite directions and in both cases regionally-specific, a generalized pathologic effect of advanced or delayed birth (within the normal range for the species) is unlikely to explain these results. Together with birth timing, other extrinsic factors also modulate cell death. For example, delivery mode (Castillo-Ruiz et al., 2018b) and the exposure to a microbiota at birth (CastilloRuiz et al., 2018a) affect the magnitude of cell death in the neonatal mouse brain. Moreover, effects are regionally specific in both cases, as in the current study. It is possible that these findings reflect regional variability in the sensitivity to factors that change at birth, such as hormones or cytokines. The hippocampus, for example, is especially sensitive to glucocorticoids (Sapolsky, 1996), so if the stress response following a delayed birth is greater than that of an on-time birth (as suggested by Rahman et al., 2017), hippocampal cell death might be affected.

Obstetric practices across the world now routinely alter human birth timing, usually to advance it. Elective cesareansections are, by definition, scheduled prior to 40 weeks gestation (Gibbons et al., 2010), and account for $>15 \%$ of the births in the US and other countries (Betrán et al., 2016). Similarly, elective labor induction is common prior to fullterm and advances birth timing (Salemi et al., 2016). The ability of birth to advance cell death could be an adaptation that reflects the importance of cell pruning for the maturation of brain circuits. However, if other neurodevelopmental events do not follow suit, as suggested by our measurements of total brain growth, cell death timing may be uncoupled from other aspects of brain development. This could be problematic, because tight temporal control is a basic feature of neural development (Durand and Raff, 2000; Rougvie, 2005; Moss, 2007), and disruptions can lead to developmental defects (Bateman and McNeill, 2004). Changes in the overall magnitude of neonatal cell death may lead to long-term changes in neuron number, as shown for effects of birth mode in mice (Castillo-Ruiz et al., 2018b). Because naturally-occurring cell death in the human brain is ongoing at birth (Lossi et al., 1998; Abrahám et al., 2001; Lavezzi et al., 2006; Abitz et al., 2007), it is possible that some of the reported effects of birth timing on child development (El Marroun et al., 2012; Abel et al., 2017; Heuvelman et al., 2018) are related to alterations in cell death, or a dissociation of cell death from other neurodevelopmental events

\section{References}

Abel K, Heuvelman H, Wicks S, Rai D, Emsley R, Gardner R, Dalman C (2017) Gestational age at birth and academic performance: Population-based cohort study. Int J Epidemiol 46:324-335.

Abitz M, Nielsen RD, Jones EG, Laursen H, Graem N, Pakkenberg B (2007) Excess of neurons in the human newborn mediodorsal thalamus compared with that of the adult. Cereb Cortex 17:2573-2578.

Abrahám H, Tornóczky T, Kosztolányi G, Seress L (2001) Cell formation in the cortical layers of the developing human cerebellum. Int J Dev Neurosci 19:53-62.
Ahern TH, Krug S, Carr AV, Murray EK, Fitzpatrick E, Bengston L, McCutcheon J, De Vries GJ, Forger NG (2013) Cell death atlas of the postnatal mouse ventral forebrain and hypothalamus: Effects of age and sex. J Comp Neurol 521:2551-2569.

Bateman JM, McNeill H (2004) Temporal control of differentiation by the insulin receptor/tor pathway in Drosophila. Cell 119:87-96.

Baulieu EE (1989) Contragestion and other clinical applications of RU 486, an antiprogesterone at the receptor. Science 245:13511357.

Betrán AP, Ye J, Moller AB, Zhang J, Gülmezoglu AM, Torloni MR (2016) The increasing trend in Caesarean section rates: Global, regional and national estimates: 1990-2014. PLoS One 11: e0148343.

Castillo-Ruiz A, Mosley M, George AJ, Mussaji LF, Fullerton EF, Ruszkowski EM, Jacobs AJ, Gewirtz AT, Chassaing B, Forger NG (2018a) The microbiota influences cell death and microglial colonization in the perinatal mouse brain. Brain Behav Immun 67:218229.

Castillo-Ruiz A, Mosley M, Jacobs AJ, Hoffiz YC, Forger NG (2018b) Birth delivery mode alters perinatal cell death in the mouse brain. Proc Natl Acad Sci USA 115:11826-11831.

Chen J, Aguilera G (2010) Vasopressin protects hippocampal neurones in culture against nutrient deprivation or glutamate-induced apoptosis. J Neuroendocrinol 22:1072-1081.

Dekkers MP, Nikoletopoulou V, Barde YA (2013) Cell biology in neuroscience: Death of developing neurons: New insights and implications for connectivity. J Cell Biol 203:385-393.

Donahue LR (2012) Gestation duration and maternal weight gain in 15 inbred mouse strains. The Jackson Laboratory. Mouse Phenome Database. Available from https://phenome.jax.org/measures/44402/ animalvalues.

Dudley DJ, Branch DW, Edwin SS, Mitchell MD (1996) Induction of preterm birth in mice by RU486. Biol Reprod 55:992-995.

Durand B, Raff M (2000) A cell-intrinsic timer that operates during oligodendrocyte development. Bioessays 22:64-71.

El Marroun H, Zeegers M, Steegers EAP, van der Ende J, Schenk JJ, Hofman A, Jaddoe VWV, Verhulst FC, Tiemeier H (2012) Post-term birth and the risk of behavioural and emotional problems in early childhood. Int J Epidemiol 41:773-781.

Estrada M, Varshney A, Ehrlich BE (2006) Elevated testosterone induces apoptosis in neuronal cells. J Biol Chem 281:25492-25501.

Evers KS, Wellmann S (2016) Arginine vasopressin and copeptin in perinatology. Front Pediatr 4:75.

Gibbons L, Belizán JM, Lauer JA, Betrán AP, Merialdi M, Althabe F (2010) The global numbers and costs of additionally needed and unnecessary caesarean sections performed per year: Overuse as a barrier to universal coverage. World Health Report 30:1-31.

Golightly E, Jabbour HN, Norman JE (2011) Endocrine immune interactions in human parturition. Mol Cell Endocrinol 335:52-59.

Grosskortenhaus R, Pearson BJ, Marusich A, Doe CQ (2005) Regulation of temporal identity transitions in Drosophila neuroblasts. Dev Cell 8:193-202.

Hammock EA (2015) Developmental perspectives on oxytocin and vasopressin. Neuropsychopharmacology 40:24-42.

Happe HK, Coulter CL, Gerety ME, Sanders JD, O'Rourke M, Bylund DB, Murrin LC (2004) Alpha-2 adrenergic receptor development in rat CNS: An autoradiographic study. Neuroscience 123:167-178.

Hashimoto H, Eto T, Endo K, Itai G, Kamisako T, Suemizu H, Ito M (2010) Comparative study of doses of exogenous progesterone administration needed to delay parturition in Jcl:MCH(ICR) mice. Exp Anim 59:521-524.

Hengartner MO (2000) The biochemistry of apoptosis. Nature 407:770-776.

Heuvelman H, Abel K, Wicks S, Gardner R, Johnstone E, Lee B, Magnusson C, Dalman C, Rai D (2018) Gestational age at birth and risk of intellectual disability without a common genetic cause. Eur J Epidemiol 33:667-678.

Hill NC, Selinger M, Ferguson J, MacKenzie IZ (1990) The placental transfer of mifepristone (RU 486) during the second trimester and 
its influence upon maternal and fetal steroid concentrations. $\mathrm{Br} \mathrm{J}$ Obstet Gynaecol 97:406-411.

Holme Nielsen C, Bladt Brandt A, Thymann T, Obelitz-Ryom K, Jiang P, Vanden Hole C, van Ginneken C, Pankratova S, Sangild PT (2018) Rapid postnatal adaptation of neurodevelopment in pigs born late preterm. Dev Neurosci 40:586-600.

Kaiser WJ, Daley-Bauer LP, Thapa RJ, Mandal P, Berger SB, Huang C, Sundararajan A, Guo H, Roback L, Speck SH, Bertin J, Gough PJ, Balachandran S, Mocarski ES (2014) RIP1 suppresses innate immune necrotic as well as apoptotic cell death during mammalian parturition. Proc Natl Acad Sci USA 111:7753-7758.

Kingsbury MA, Bilbo SD (2019) The inflammatory event of birth: How oxytocin signaling may guide the development of the brain and gastrointestinal system. Front Neuroendocrinol 55:100794.

Lagercrantz H, Slotkin TA (1986) The "stress" of being born. Sci Am 254:100-107.

Lavezzi AM, Ottaviani G, Terni L, Matturri L (2006) Histological and biological developmental characterization of the human cerebellar cortex. Int J Dev Neurosci 24:365-371.

Lossi L, Zagzag D, Greco MA, Merighi A (1998) Apoptosis of undifferentiated progenitors and granule cell precursors in the postnatal human cerebellar cortex correlates with expression of BCL-2, ICE, and CPP32 proteins. J Comp Neurol 399:359-372.

Mann CL, Hughes FM Jr, Cidlowski JA (2000) Delineation of the signaling pathways involved in glucocorticoid-induced and spontaneous apoptosis of rat thymocytes. Endocrinology 141:528-538.

Mitchell BF, Taggart MJ (2009) Are animal models relevant to key aspects of human parturition? Am J Physiol Regul Integr Comp Physiol 297:R525-R545.

Morrissy S, Xu B, Aguilar D, Zhang J, Chen QM (2010) Inhibition of apoptosis by progesterone in cardiomyocytes. Aging Cell 9:799809.

Mosley M, Shah C, Morse KA, Miloro SA, Holmes MM, Ahern TH, Forger NG (2017) Patterns of cell death in the perinatal mouse forebrain. J Comp Neurol 525:47-64.

Moss EG (2007) Heterochronic genes and the nature of developmental time. Curr Biol 17:R425-R434.

Mulholland PJ, Stepanyan TD, Self RL, Hensley AK, Harris BR, Kowalski A, Littleton JM, Prendergast MA (2005) Corticosterone and dexamethasone potentiate cytotoxicity associated with oxygen-glucose deprivation in organotypic cerebellar slice cultures. Neuroscience 136:259-267.

Murray SA, Morgan JL, Kane C, Sharma Y, Heffner CS, Lake J, Donahue LR (2010) Mouse gestation length is genetically determined. PLoS One 5:e12418.

Noguchi KK, Walls KC, Wozniak DF, Olney JW, Roth KA, Farber NB (2008) Acute neonatal glucocorticoid exposure produces selective and rapid cerebellar neural progenitor cell apoptotic death. Cell Death Differ 15:1582-1592.

Noh JS, Kim EY, Kang JS, Kim HR, Oh YJ, Gwag BJ (1999) Neurotoxic and neuroprotective actions of catecholamines in cortical neurons. Exp Neurol 159:217-224.

Oppenheim RW (1991) Cell death during development of the nervous system. Annu Rev Neurosci 14:453-501.

Osman I, Young A, Ledingham MA, Thomson AJ, Jordan F, Greer IA, Norman JE (2003) Leukocyte density and pro-inflammatory cytokine expression in human fetal membranes, decidua, cervix and myometrium before and during labour at term. Mol Hum Reprod 9:41-45.

Paxinos G, Halliday G, Watson C, Koutcherov Y, Wang HQ (2007) Atlas of the developing mouse brain: E17.5, P0, and P6. San Diego: Elsevier.

Porter AG, Jänicke RU (1999) Emerging roles of caspase-3 in apoptosis. Cell Death Differ 6:99-104.

Quadros PS, Pfau JL, Wagner CK (2007) Distribution of progesterone receptor immunoreactivity in the fetal and neonatal rat forebrain. $J$ Comp Neurol 504:42-56.

Quadros PS, Schlueter LJ, Wagner CK (2008) Distribution of progesterone receptor immunoreactivity in the midbrain and hindbrain of postnatal rats. Dev Neurobiol 68:1378-1390.

Rahman A, Cahill LS, Zhou YQ, Hoggarth J, Rennie MY, Seed M, Macgowan CK, Kingdom JC (2017) A mouse model of antepartum stillbirth. Am J Obstet Gynecol 217:443.e1-443.e11.

Ratajczak CK, Muglia LJ (2008) Insights into parturition biology from genetically altered mice. Pediatr Res 64:581-589.

Rougvie AE (2005) Intrinsic and extrinsic regulators of developmental timing: From miRNAs to nutritional cues. Development 132:37873798.

Salemi JL, Pathak EB, Salihu HM (2016) Infant outcomes after elective early-term delivery compared with expectant management. Obstet Gynecol 127:657-666.

Sapolsky RM (1996) Stress, glucocorticoids, and damage to the nervous system: The current state of confusion. Stress 1:1-19.

Southwell DG, Paredes MF, Galvao RP, Jones DL, Froemke RC, Sebe JY, Alfaro-Cervello C, Tang Y, Garcia-Verdugo JM, Rubenstein JL, Baraban SC, Alvarez-Buylla A (2012) Intrinsically determined cell death of developing cortical interneurons. Nature 491:109-113.

Srinivasan A, Roth KA, Sayers RO, Shindler KS, Wong AM, Fritz LC, Tomaselli KJ (1998) In situ immunodetection of activated caspase3 in apoptotic neurons in the developing nervous system. Cell Death Differ 5:1004-1016.

Toda T, Homma D, Tokuoka H, Hayakawa I, Sugimoto Y, Ichinose H, Kawasaki H (2013) Birth regulates the initiation of sensory map formation through serotonin signaling. Dev Cell 27:32-46.

Walker JC, Smolders MA, Gemen EF, Antonius TA, Leuvenink J, de Vries $E$ (2011) Development of lymphocyte subpopulations in preterm infants. Scand J Immunol 73:53-58.

Waters EM, Simerly RB (2009) Estrogen induces caspase-dependent cell death during hypothalamic development. J Neurosci 29:97149718.

Wolf JP, Chillik CF, Itskovitz J, Weyman D, Anderson TL, Ulmann A, Baulieu EE, Hodgen GD (1988) Transplacental passage of a progesterone antagonist in monkeys. Am J Obstet Gynecol 159:238242.

Yi SJ, Masters JN, Baram TZ (1994) Glucocorticoid receptor mRNA ontogeny in the fetal and postnatal rat forebrain. Mol Cell Neurosci 5:385-393.

Zuloaga DG, Carbone DL, Hiroi R, Chong DL, Handa RJ (2011) Dexamethasone induces apoptosis in the developing rat amygdala in an age-, region-, and sex-specific manner. Neuroscience 199:535-547. 